\title{
Atypical presentation of nasopharyngeal carcinoma
}

\begin{abstract}
Nasopharyngeal Carcinoma (NPC) is a rare neoplasm which is associated with Epstein Barr Virus (EBV). We report a case of an 18 year-old African American male patient with no significant past medical history, presenting with a unilateral neck mass. Imaging and biopsy confirmed a neoplasm, diagnosed as nasopharyngeal carcinoma. Our case is remarkable as NPC is perhaps most common in China where annual incidence is 25 cases per 100,000 compared to the US, with an annual incidence of 0.5 cases per 100,000. Our patient is African American and had never been outside the country. Furthermore, he is young, while most cases of NPC happen in the elderly. While age and race were atypical, our patient was both a smoker and EBV positive. Finally, as a stage 2 NPC he received chemo radiotherapy and has thus far responded well.
\end{abstract}

Keywords: nasopharyngeal, chemo radiotherapy, emergency room, nasopharynx, neoplasm
Volume 2 Issue 4 - 2016

\author{
Murad Baba,' Dominguez LW,' Amit Patel ${ }^{2}$ \\ 'Department of Internal Medicine, USA \\ ${ }^{2}$ Department of Hematology/Oncology, USA
}

Correspondence: Murad Baba, Department of Internal Medicine, Jersey City Medical Center, 355 Grand Street, Jersey City, USA, 07302, Fax +2019152219,

Emailmuurad@hotmail.com

Received: April 25, 2016| Published: June 16, 2016
Abbreviations: NPC, nasopharyngeal carcinoma; EBV, epstein barr virus; EBERs, EBV encoded RNAs; IMRT, intensity modulated radiation therapy; $\mathrm{CT}$, computed tomography; IMRT, intensity modulated radiation therapy; GTV, gross tumor volume

\section{Introduction}

Nasopharyngeal carcinoma (NPC) is a rare neoplasm which is associated with Epstein Barr virus (EBV). Overall prognosis has improved significantly over the past three decades because of advances in disease management, diagnostic imaging, radiotherapy technology, and broader application of systemic therapy.

\section{Case description}

A 18 year-old African American male, smoker with no significant past medical history, presented to the Emergency Room (ER) complaining of unilateral neck pain, he was sent home on pain killers and muscle relaxant. The following two months his pain did not get better, he developed sore throat and was seen by his PMD who found a neck lymph node enlargement and prescribed him antibiotics. On his second visit to the ER a Computed Tomography (CT) scan of the neck was ordered and showed a nasopharyngeal mass. The patient denied fever, night sweats, weight loss or any other complaints. He had no history of trauma, surgery. He smokes cigarettes, drinks alcohol occasionally. Physical examination showed right side LN enlargement in the neck around $2 \times 2 \mathrm{~cm}$, firm in consistency.

His laboratory workup did not show any abnormality. Imaging studies including a Neck CT scan demonstrated enhancing lobulated lesion along the right tonsillar/peritonsillar region extending up to the nasopharynx measuring $6 \times 4 \mathrm{~cm}$, associated with mild displacement of the internal carotid artery and internal jugular vein postero laterally. The lesion wraps near circumferentially around these vessels. In addition to mildly enlarged right level two lymph nodes, with the largest measuring $2 \mathrm{~cm}$. Ultrasound guided biopsy of the occipital neck LN was arranged, the pathology result identified metastatic squamous nasopharyngeal carcinoma. Immunohistochemical analysis and in situ hybridization of the occipital lymph node further confirmed the presence of EBV Encoded RNAs (EBERs) indicating EBERs expression in tumor cells, as well as positivity for CK 5/6 and P40, as demonstrated in (Figure 1).

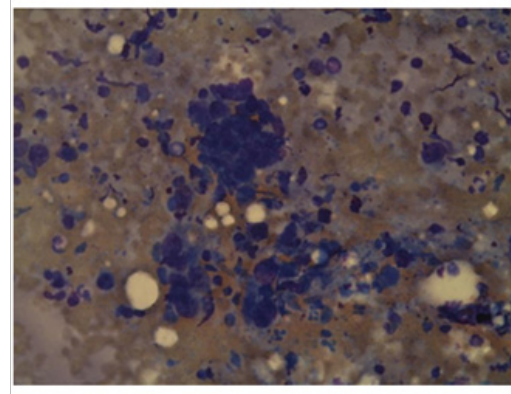

(A)

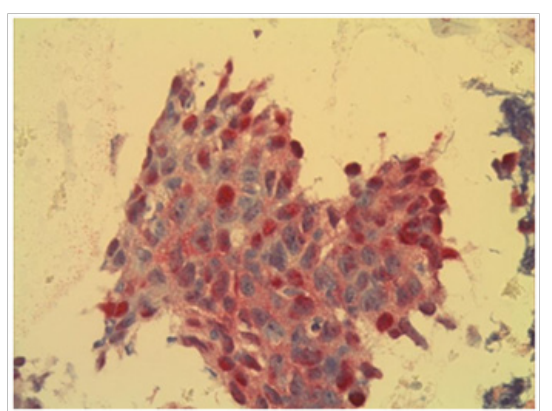

(B)

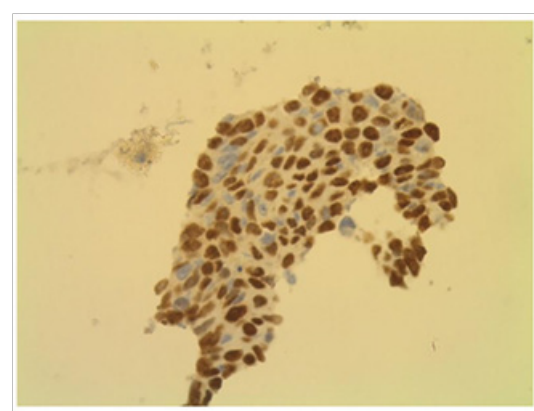

(C)

Figure I Fine NeedleAspiration (FNA) of the posterior neck lymph node. (A) Hematoxylin and eosin (H and E) stained section shows tumor cells characteristic of NPC cells.(B) Immunohistochemical analysis and in situ hybridization of the lymph node shows the expression of Epstein-Barr virus Encoded RNAs (EBERs) in tumor cells. (C) Immunohistochemical stain shows squamous epithelium with P40 expression.

Other radiographic studies, including chest radiography and abdominal CT scan showed no distant metastasis. PET/CT scan was done before starting treatment and showed the mass in the right side of the nasopharynx with bilateral posterior cervical LNs right more than the left, and no distant metastasis. Accordingly, the patient was diagnosed as having T2N1M0 stage 2 non-keratinizing NPC. 
The patient received chemotherapy with Cisplatin $\left(40 \mathrm{mg} / \mathrm{m}^{2}\right)$ weekly for 7 weeks along with Intensity Modulated Radiation Therapy (IMRT): a total dose of 70Gy to the Gross Tumor Volume (GTV), all doses were given in 35 treatments, over 58days. The whole course of treatment was completed with no disruptions. Follow up 8 weeks after completion of radiotherapy with PET/CT scan showed that the primary lesion and lesions in the cervical lymph nodes are no longer seen. At 6-months follow up, he appears to be doing well with no clinical or radiological evidence of the disease.

\section{Discussion}

\section{Epidemiology}

Nasopharyngeal carcinoma (NPC) has an annual incidence of 86,000 cases, and is responsible for 50,000 fatalities worldwide. ${ }^{1}$ Like most cancers, the risk for NPC increases with age, peaking in the $50 \mathrm{~s} .{ }^{2}$ There is also a geographic distribution, as Southeast Asia, the Middle East and North Africa have prevalence 10-50times higher than the United States. ${ }^{3}$

\section{Clinical presentation}

Symptomatically NPC most often presents with nasal and ear complaints from sinus congestion and epistaxis, to earaches and tinnitus. Physical exam classically reveals a neck mass or masses, and cranial nerve involvement is not uncommon. Unfortunately, nasopharyngeal carcinoma is highly aggressive, spreading mostly through lymphatics. Accordingly, $75-90 \%$ of new cases are already metastatic, with $50 \%$ presenting with bilateral neck masses. ${ }^{4}$ Around $5-10 \%$ of cases have distant metastasis, most commonly involving the bone followed by liver then the lungs. ${ }^{4}$ As with most cancers, distant lymph node metastasis is a poor prognostic indicator. ${ }^{5}$

\section{Etiology}

Etiologically, NPC has genetic, environmental, and infectious components. One case control study found that first degree relatives are seven times more likely to acquire the disease when a family member has the cancer. ${ }^{6}$ Environmentally, multiple studies have linked smoking to NPC. ${ }^{7,8}$ However, perhaps the most notable agent in the development of NPC is EBV infection. ${ }^{9}$ This is notable as $\mathrm{EBV}$; along with Hepatitis $\mathrm{C}$ are some of the few pathogens directly implicated in neoplastic transformation.

EBV is known to infect most adults worldwide mostly asymptomatic, usually with lifelong persistence. The virus is associated with rare malignant transformations in lymphoid cells or epithelial tissue; malignancies linked to EBV infection include nasopharyngeal carcinoma, Burkit Lymphoma, Hodgkin Lymphoma and tumors in HIV infected patients. Numerous studies have shown diagnostic and prognostic utility of anti-EBV for nasopharyngeal carcinoma. ${ }^{8}$ EBV is capable of transforming B cells but does not routinely display a cytopathic effect in cell culture, unlike other viruses as HSV and CMV. Persistent EBV infection may be the result of viral evasion strategies to host immune responses like most tumors, definitive diagnosis requires biopsy. Careful examination of cranial nerves is important to assess severity and spread. MRI is the imaging modality of choice, as it is superior for distinguishing soft-tissue structures in the retropharyngeal spaces..$^{5}$

As NPC presents late stage, screening can be an important tool. While the cancer has extremely high prevalence in certain geographic regions, it also has a strong association with EBV. As a result, some research has focused on using EBV as a screening tool for NPC.
Multiple serological markers, targeting EBV antibodies have been studied to detect infection and predict NPC risk. ${ }^{4}$ Unfortunately, antibody positivity alone has shown poor sensitivity, as their presence can precede the development of malignancy by up to 10years. ${ }^{10}$ However, EBV detection through PCR has shown much more promise, with $99 \%$ sensitivity in detecting cases of NPC. ${ }^{11}$ While EBV positivity portends NPC, the extent of viral infection has also been shown to be prognostic. Baseline levels of EBV DNA can actually be used to stratify prognosis, with higher titers indicating greater disease severity and poorer outcome. Furthermore, studies have shown declining EBV DNA levels as a proxy measure for successful treatment. As a result, initial and serial EBV DNA levels are crucial in plotting the course of the disease and efficacy of treatment. ${ }^{12}$

\section{Treatment}

Staging follows the classic TMN paradigm. Intensity Modulated Radiation Therapy (IMRT) is the mainstay for treatment. ${ }^{13}$ As nasopharyngeal carcinoma has a propensity for fast bilateral metastasis, all patients receive bilateral radiation. ${ }^{14}$ When it comes to N0 staged carcinomas, one study suggests success without having to use radiation. ${ }^{15}$ When detected in stage 1 , radiation monotherapy can produce a 5year survival rate of over $90 \% .{ }^{16}$ However, it should be noted that these patients are not typically enrolled in studies using chemotherapy for Stage 1 and thus insufficient evidence exists for adjuvant efficacy. ${ }^{17}$ For stages 2 and higher, radiation must be supplemented with chemotherapy. Prompt combination of radiation with weekly cisplatin achieved a $94.5 \%$ 5year survival rate in stage 2 patients. ${ }^{18}$ It should be noted that the addition of cisplatin significantly increased the rate of leukopenia and GI upset, as would be expected. ${ }^{18}$

Stage 3 and 4 require combination therapy as well, however specific regimens, and thus specific survival rates, are yet to be agreed upon. A recently released trial looked at 1267 patients, comparing radiation monotherapy versus chemo radiotherapy, and there was a significant survival benefit to adding chemotherapy to radiotherapy alone. ${ }^{19}$ Unfortunately, as this was a literature review, a single best chemotherapy was not illuminated. Furthermore, it was not possible to differentiate the efficacy of adjuvant versus concurrent chemotherapy when undergoing RT.

As mentioned, higher titers of EBV DNA portend a poorer prognosis, ${ }^{20}$ and falling levels of EBV DNA indicate successful treatment. ${ }^{12}$ As such, serial EBV DNA levels taken before, during and after treatment are essential in plotting disease progression and surveillance. Follow up surveillance should include exam of the nasopharynx, neck and cranial nerves, especially as nasopharyngeal carcinoma has been known to recur much later than other head and neck cancers, and in variable locations, especially in bone.

\section{Conclusion}

We report a case of an 18year old African American male patient with no past medical history, presenting with a unilateral neck mass. Imaging and biopsy confirmed a neoplasm, diagnosed as nasopharyngeal carcinoma. Our case is remarkable as NPC is perhaps most common in China where annual incidence is 25 cases per $100,000,{ }^{3}$ compared to the US, with an annual incidence of 0.5 cases per $100,000 .{ }^{2}$ Our patient is African American, and had never been outside the country. Furthermore, he is young, while most cases of NPC happen in the elderly. While age and race were atypical, our patient was both a smoker and EBV positive. Finally, as a stage 2 NPC he received chemo radiotherapy and has thus far responded well with near complete resolution of the nasopharyngeal mass. 


\section{Acknowledgements}

None.

\section{Conflict of interest}

The author declares no conflict of interest.

\section{References}

1. Ferlay J, Soerjomataram I, Dikshit R, et al. Cancer incidence and mortality worldwide: sources, methods and major patterns in GLOBOCAN 2012. Int J Cancer. 2015;136(5):E359-E386.

2. Chang ET, Adami HO. The enigmatic epidemiology of nasopharyngeal carcinoma. Cancer Epidemiol Biomarkers Prev. 2006;15(10):17651777 .

3. Barnes L, Eveson JW, Reichart P, et al. Pathology and genetics of head and neck tumors. In: Leon Barnes, et al. editors. World Health Organization Classifications of Tumors. Lyon, France: IARC Press; 2005.

4. Altun M, Fandi A, Dupuis O, et al. Undifferentiated nasopharyngeal cancer (UCNT): Current diagnostic and therapeutic aspects. Int J Radiat Oncol Biol Phys. 1995;32(3):859-877.

5. Liao XB, Mao YP, Liu LZ, et al. How does magnetic resonance imaging influence staging according to AJCC staging system for nasopharyngeal carcinoma compared with computed tomography? Int J Radiat Oncol Biol Phys. 2008;72(5):1368-1377.

6. Ung A, Chen CJ, Levine PH, et al. Familial and sporadic cases of nasopharyngeal carcinoma in Taiwan. Anticancer Res. 1999;19(1B):661665 .

7. Xu FH, Xiong D, Xu YF, et al. An epidemiological and molecular study of the relationship between smoking, risk of nasopharyngeal carcinoma, and Epstein-Barr virus activation. J Natl Cancer Inst. 2012;104(18):1396-1410.

8. Hsu WL, Chen JY, Chien YC, et al. Independent effect of EBV and cigarette smoking on nasopharyngeal carcinoma: a 20-year followup study on 9,622 males without family history in Taiwan. Cancer Epidemiol Biomarkers Prev. 2009;18(4):1218-1226.

9. Raghupathy R, Hui EP, Chan AT. Epstein-Barr virus as a paradigm in nasopharyngeal cancer: from lab to clinic. Am Soc Clin Oncol Educ Book. 2014;149-153.
10. Chua DT, Ji M, Zong Y, Chan K, et al. Screening of nasopharyngeal carcinoma by serology and nasopharyngoscopy and treatment outcome in endemic region. ASCO Annual Meeting. 2009;7(15S):6029.

11. Leung SF, Tam JS, Chan AT, et al. Improved accuracy of detection of nasopharyngeal carcinoma by combined application of circulating Epstein-Barr virus DNA and anti-Epstein-Barr viral capsid antigen IgA antibody. Clin Chem. 2004;50(2):339-345.

12. Hui EP, Ma BB, Chan $\mathrm{KC}$, et al. Clinical utility of plasma EpsteinBarr virus DNA and ERCC1 single nucleotide polymorphism in nasopharyngeal carcinoma. Cancer. 2015;121(16):2720-2729.

13. Peng G, Wang T, Yang KY, et al. A prospective, randomized study comparing outcomes and toxicities of intensity-modulated radiotherapy vs. conventional two-dimensional radiotherapy for the treatment of nasopharyngeal carcinoma. Radiother Oncol. 2012;104(3):286-293.

14. Lee AW, Poon YF, Foo W, et al. Retrospective analysis of 5037 patients with nasopharyngeal carcinoma treated during 1976-1985: overall survival and patterns of failure. Int J Radiat Oncol Biol Phys. 1992;23:261-270.

15. Li JG, Yuan X, Zhang LL, et al. A randomized clinical trial comparing prophylactic upper versus whole-neck irradiation in the treatment of patients with node-negative nasopharyngeal carcinoma. Cancer. 2013;119:3170-3176.

16. Lee AW, Sze WM, Au JS, et al. Treatment results for nasopharyngeal carcinoma in the modern era: the Hong Kong experience. Int $J$ Radiat Oncol Biol Phys. 2005;61(4):1107-1116.

17. Chua DT, Ma J, Sham JS, et al. Improvement of survival after addition of induction chemotherapy to radiotherapy in patients with early-stage nasopharyngeal carcinoma: Subgroup analysis of two Phase III trials. Int J Radiat Oncol Biol Phys. 206;65(5):1300-1306.

18. Chen QY, Wen YF, Guo L, et al. Concurrent chemo radiotherapy vs radiotherapy alone in stage II nasopharyngeal carcinoma: phase III randomized trial. J Natl Cancer Inst. 2011;103(23):1761-1770.

19. Blanchard P, Lee A, Marguet S, et al. Chemotherapy and radiotherapy in nasopharyngeal carcinoma: an update of the MAC-NPC meta-analysis. Lancet Oncol. 2015;16(6):645-655.

20. Lin JC, Wang WY, Chen KY, et al. Quantification of plasma Epstein-Barr virus DNA in patients with advanced nasopharyngeal carcinoma. $N$ Engl J Med. 2004;350(24):2461-2470. 